\title{
DESARROLLO TURÍSTICO Y SU RELACIÓN CON EL TRANSPORTE
}

\author{
Ninoska Elena Viloria Cedeño \\ Universidad Experimental Nacional Libertador \\ Venezuela. \\ nvc26@hotmail.com.
}

\section{RESUMEN}

En la medida que la tecnociencia avanza y mejora, crece el sector turismo, se persigue con el presente artículo analizar la importancia e interrelación del avance tecnológico-científico del transporte y su aporte al turismo, el soporte teórico se fundamentó en investigadores como Hunziker y Krapf (1942), Leiper (1979), Nuñez (2007), Organización mundial de turismo (OMT, 2011). Metodológicamente la investigación se enfocó documental, puesto que Los medios de transporte han cooperado notablemente con el crecimiento y desarrollo del sector turístico; porque el hombre ha buscado desplazarse de un lugar a otro y ha contado con los avances de la ciencia y tecnología. Como conclusión se tiene que en la medida que se han mejorado o perfeccionado los medios de transporte la demanda turística ha crecido vertiginosamente. En el mundo de los viajes, los intercambios culturales, sociales, económicos, políticos, se producen porque existen los medios de transporte.

Palabras claves: Turismo, medios de transportación, tecnología, relaciones. 


\title{
TOURIST DEVELOPMENT AND ITS RELATION TO TRANSPORTATION
}

\author{
Ninoska Elena Viloria Cedeño \\ Universidad Experimental Nacional Libertador \\ Venezuela. \\ nvc26@hotmail.com.
}

\begin{abstract}
As techno-science advances and it improves, tourism grows. This article pursues to analyze the importance and interrelation of the technological-scientific advancement of transportation and its contribution to tourism. The theoretical support was based on investigators such as Hunziker and Krapf (1942), Leiper (1979), Núñez (2007), World Tourism Organization (OMT, 2011). Methodologically, the investigation was documentary, since the means of transportation have cooperated notably with the growth and development of the tourism sector, because man has searched for moving from one place to another and he has had the advances of science and technology. As a conclusion, as the means of transportation have been improved or perfected the tourist demand has grown vertiginously. In the world of trips, cultural, social, economic and political exchanges take place because means of transportation exist.
\end{abstract}

Key words: Tourism, transportation means, technology, relationships. 


\section{INTRODUCCIÓN}

El impacto que ha representado para la sociedad los medios de transporte es de tal magnitud, que para poder efectuar cualquier tipo de desplazamiento se requiere de éstos. El hombre desde su nacimiento ha contado con algún mecanismo para moverse de un lado a otro, iniciando una cadena que comienza en el vientre, en los brazos de la madre, posteriormente gateando y por último caminando. De esta manera, fue progresando en su intento por recorrer distancias en el menor tiempo posible y efectuándola a través de sus extremidades inferiores; tomando en cuenta estos factores espacio - tiempo, se hacían de difícil ejecución para determinadas épocas. Esto le permitió al hombre estudiar o analizar como mejorar las condiciones de espacio - tiempo, es aquí donde surge la importancia de la tecnociencia. El primer medio de transporte después del humano, es el de los animales y éste a su vez sirvió para transportar estructuras de madera o metálicas como carretas, carrozas, entre otros; de esta manera fue perfeccionando los diseños hasta llegar a diseñar los ferrocarriles, el carro, entre otros.

Después de este desarrollo del transporte terrestre, el hombre analizó que con el carro no podía pasar el agua en sitios profundos, es decir, mares, océanos, lagos, entre otros; entonces desarrolla las primeras canoas, y así hasta llegar hoy en día a la construcción de barcos, transbordadores y otros medios para transportarse. El otro medio de transporte fue el aéreo, en el cual la inquietud de semejar a la naturaleza en especial a las aves, fue lo que inspiró a Leonardo Da Vinci hacer el primer bosquejo de un helicóptero y partiendo de ésto, es cuando comienza la revolución del transporte aéreo a través de naves como helicópteros, aviones, avionetas. Por último, se tiene el transporte espacial el cual de muy reciente data ha permitido que en los actuales momentos el poder de traspasar los límites del planeta tierra sea una realidad.

Como se puede notar detrás de todo estos progresos del transporte está la tecnología y la ciencia que han contribuido en el desarrollo de los medios de transportación. La tecnociencia ha perfeccionado cada uno de los medios de transporte, es el que ha permitido mejorar el factor espacio - tiempo, economía, confort y la conveniencia de escoger el más adecuado para trasladarse. Lo trascendente de este avance tecnológico-científico es que ha coadyuvado a relacionar a los seres humanos, a efectuar intercambios culturales, a pesar que saliendo de los límites de la estratosfera se puede mantener contacto con otros seres, sea esto especulativo o no. Esto hace que cada vez más en el campo tecnológico el 
transporte sea un pilar fundamental como lo es hoy en día las telecomunicaciones, aún cuando se tenga diferentes comunicaciones radiales, escritas e incluso Internet, el hombre por naturaleza siempre buscará desplazarse, por razones de negocios, salud, deportivas, de esparcimiento, entre otros aspectos.

\section{RELACIÓN DEL TRANSPORTE CON EL SECTOR TURISMO}

Para comenzar a determinar las relaciones y la importancia del transporte con el sector turismo, es conveniente preguntarse primero ¿Qué es turismo? y ¿Por qué el transporte condiciona el Turismo?; estas interrogantes requieren ser aclaradas para ir compenetrándose con el tema y posteriormente ubicar las relaciones. Al turismo le han asignado varias definiciones que a continuación se dan a conocer: "El Turismo es un fenómeno social que consiste en el desplazamiento voluntario y temporal de individuos o grupos de personas que fundamentalmente por motivos de recreación, descanso, cultura o salud, se trasladan de un lugar de residencia habitual a otro en el que o ejercen alguna actividad lucrativa ni remunerada, generando múltiples interrelaciones de suma importancia económica y cultural" (Padilla, 1980:74). "El turismo es un conjunto bien definido de relaciones, servicios e instalaciones que se generan en virtud de ciertos desplazamientos humanos" (Cuervo, 1967:29). Para dar otra referencia se tiene también el turismo desde un punto de vista de la teoría general de los sistemas como es: "un sistema abierto, de cinco elementos, interactuando en un amplio medio ambiente. Siendo estos elementos: uno dinámico, el turista; tres geográficos: la región generadora, la ruta de tránsito y la región de destino, y un elemento económico, la industria turística" (Leiper, 1979:309). Como se puede observar en las definiciones antes presentadas la palabra desplazamiento está como referencia básica para efectuar turismo, por lo tanto la finalidad de los medios de transporte dentro del sistema turístico es la de permitir el desplazamiento del turista, esto hace que estos medios permitan tener acceso al lugar en el cual se ubican o localizan los atractivos turísticos.

Asimismo, los fundadores de la escuela humanística definieron el turismo "un conjunto de relaciones y fenómenos producidos por el desplazamiento y la permanencia temporal de personas fuera de su lugar de residencia habitual, sin que sean motivados por el lucro" (Hunziker y Krapf, 1942:5). 
Generalizando las definiciones emitidas por los autores antes citados, vinculan la relación tiempo -espacio básicamente y desde este punto de vista intrínsecamente en el término desplazamiento esta el medio del transporte. De igual manera, presentan el turismo como un fenómeno, conjunto de relaciones e incluso como sistema; pero analizando cada una de las acepciones tenemos por ejemplo el término fenómeno, profundizando como tal, el fenómeno lo hace el transporte, sin el desplazamiento no hay viaje, puede existir pero caminando, quizás para recorrer distancias cortas o largas, sin importar el tiempo sería el caminar suficiente; pero en el mundo actual eso no funcionaría y no existiría el turismo o se haría en cada país en donde se reside y cada vez el círculo de desplazamiento sería más corto.

Como el término relación, de nada sirve tener instalaciones, servicios, infraestructuras si no hay medios para desplazarse y estar en el sitio deseado, sólo por un momento se penetra al mundo de la fantasía y se imagina un atractivo turístico, en donde se satisfagan las necesidades del turista y no se contara con transporte, surge la siguiente interrogante ¿ De qué sirve un atractivo turístico, si no hay manera de llegar a ello, considerando que estén dadas las condiciones humanas?. Tanto Cuervo (ob.cit) como Hunziker y Krapf (ob.cit) manejan criterios similares, y sigue estando presente en sus posturas el medio de transporte cuando plantean el desplazamiento.

Por último el término sistema, entendiendo la definición de éste como criterio básico un conjunto de partes para formar un todo, acá se hace referencia de manera general, sin detallar otras definiciones; si el transporte es parte de un todo, y desaparece pierde sentido ese todo, por lo tanto, el medio de desplazamiento anula o cierra las posibilidades de que el sistema se mantenga. Los transportes sean terrestres, acuáticos, aéreos, espaciales forman parte de la actividad turística porque es la que permite interrelacionarse con cada uno de los integrantes básicos de la industria como son: alojamientos, centros de atractivos turísticos, agencias de viaje, entre otros; y esto a su vez conforman el mundo del turismo.

En el campo terrestre se dio inicio a través de los pies y hoy en día pasando desde un automóvil, hasta contar con autobuses llamados Buscamas, trolebuses e incluso medios de transporte turístico más específicos del área que ya existen en varios países del mundo y sin dejar de tomar en cuenta los ferrocarriles que cada vez, sus velocidades son mayores, más amplios y con mayor comodidad; sin incluir en este tema del medio de 
transporte, el campo turístico - deportivo donde los transportes son básicos para muchas actividades que requieren desplazamientos en unidades con mecanismos especializados.

Históricamente la relación turismo-transporte parte desde el siglo XIX cuando los ferrocarriles surgen como medio de transporte básicamente en Europa y Estados Unidos, los cuales eran utilizados para asistir a los museos y visitar los monumentos por razones de esparcimiento, recreación. Seguidamente, surgen los primeros vehículos de combustión interna como medio de desplazamiento y se convierten en el transcurrir del tiempo uno de los transporte con características turísticas por excelencia, puesto que es un medio de transporte no regular, es decir, no tiene ni horario, ni ruta establecida si el mismo no pertenece a ninguna línea de transporte en particular.

En cuanto al transporte acuático se comenzó con canoas, balsas y actualmente se tienen barcos -cruceros, a los que se le llama "ciudades acuáticas", porque tienen todo el confort en el interior y exterior de las naves, es decir, realizar en el agua (mares, océanos) lo que se puede hacer en tierra. Los primeros cruceros zarparon por las rutas turísticas de Europa como es el caso de las rutas en el Mediterráneo; entre los cruceros famosos de la época se tienen el Titanic, Mauritania y el Lucitania. Asimismo, cuando se construyeron los ferry, los car-ferry (pasajeros y automóviles) y los hoovercraf naves con utilidad turística. Los grandes transbordadores que anexados a los cruceros incrementan cada día el interés de los turistas por viajar por mares y océanos.

Desde el punto de vista de transporte aéreo, después de la segunda guerra mundial se inaugura en el año 1944 la organización encarga de regular y sistematizar los vuelos comerciales I.A.T.A (Asociación internacional de Transporte Aéreo) y sucesivamente se fueron construyendo aviones con características aéreas que permitieran mejorar el desplazamiento de los pasajeros.

El transporte aéreo comercial surge en Francia con los aviones para pasajeros Aérospatiale № 262 y Frégate para 29 pasajeros en el año 1962 para la aerolínea Air Inter, luego el Aérospatiale SE 210 Caravelle para recorridos medios y capacidad para 104 a 139 pasajeros en el año 1959 y fueron adquirido por la línea aérea Air France. Las compañías aéreas francesas, inglesas y alemanas conforman un consorcio para construir los Airbus, sin embargo con el tiempo se retiraron los ingleses y se incorpora España para iniciar los 
vuelos en el año 1972. El Bac/Aérospatiale Concorde 01 para 128 pasajeros vuela en el año 1971 la fabricación fue una alianza entre Francia e Inglaterra y se construyeron 16 aviones. En el caso de los Boeing se inicio con un modelo 367-80 con capacidad entre 90 y 167 pasajeros su primer vuelo fue en el año 1954 y los modelos 707-120 y 720 comenzaron en el año 1957. La Boeing continuó su fabricación con los modelos 707-320, 707-420 (1962), 727 (1963), 737 (1967), 747 para 363 a 490 pasajeros (1970) inaugurado por la línea Pan American Nueva York - Londres. Por último surgen otros aviones de menor capacidad de pasajeros como Antonov An-22,24,26 (1963) Canadair 400 (1959); Convair-Liner (1967) 240, 340, 440, 580,600, 640; los Convair 880, 990 (1960); los Curtiss C-46 (1940); los Douglas DC-3,DC-4,DC-6,DC-7 (1936) entre otros. (Taylor \& Swanborough,1977: 5-18).

El transporte es un factor determinante del producto turístico, "representa el medio de llegar al destino, es un medio necesario para los desplazamientos dentro del destino visitado y en un número reducido de casos, la propia atracción o actividad turística" (Cooper, Fletcher, et.al, 2007: 544). En otro orden de ideas, se tiene también el impacto que puede generar la ausencia o disminución de cualquier medio de transportación a los sitios turísticos, se tiene el caso cuando suceden accidentes aéreos, ejemplo lo acontecido el 11 de septiembre del 2001 en los Estados Unidos, en el cual fueron suspendidos varios vuelos desde otros continentes hacia éste país y viceversa; influyendo notablemente en la baja turística de la época. Cuando por razones climáticas, bélicas, entre otras situaciones impiden el acceso a los atractivos turísticos, puesto que en algunos casos la transportación se obstaculiza o se suspende.

Las relaciones del transporte con el sector turismo son ineluctables, es por esto que su conexión es determinante o es un condicionante importantísimo, porque el turismo es inseparable de viajar y de esta actividad depende. Entonces el medio de transportación tiene conexión con las siguientes empresas de turismo: 
Figura $\mathbf{N}^{\mathbf{0}} \mathbf{1}$ Esquema de la relación medio de transporte y los demás facilidades que conforman el producto turístico

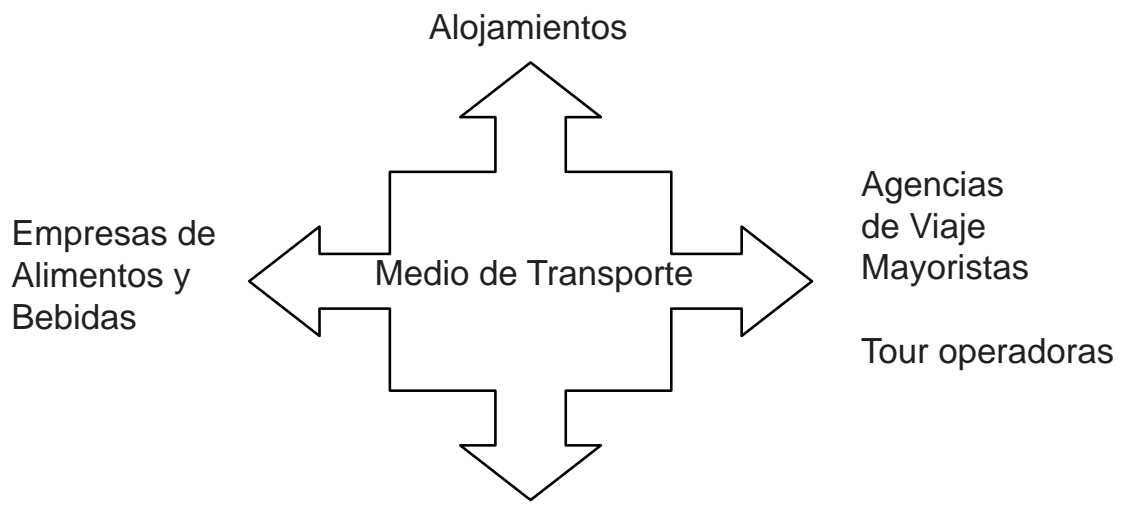

Centros de Recreación Turística

Fuente: Los Autores (2011)

Esto ha permitido un relevante impacto de crecimiento y de desarrollo del turismo; para cada medio de transportación sea público o privado se busca el confort, lo económico, lo rápido y el ser humano se ha encargado de investigar como lograr que el turista se sienta con agrado al viajar. Las investigaciones están referidas desde el aspecto económico, hasta llegar a satisfacer necesidades fáciles de realizar en tierra, pero difíciles en el mar, aire e incluso espacial. Es por estas razones antes expuesta la relación transporte y turismo es un binomio inseparable para que se pueda lograr que el (los) turista (s) puedan realizar las actividades correspondiente; por lo tanto los medios de transportación han contribuido de manera sustancial al turismo.

Se ha planteado la relación del transporte para el turismo, sin detallar en actividades turísticas que dependen de los medios de transportación no exclusivamente, pero que contribuye; se tiene el caso deportivo, puesto que numerosos eventos e incluso destinos turísticos representan un atractivo por la diversidad de usos de medios de transporte para el desplazamiento. Las primeras referencias deportivas turísticas con desplazamiento se 
dieron en Grecia entre los siglos VIII- VI a.c. con los juegos olímpicos (se crearon en el año 776) y sucesivamente esto permitió relacionar el deporte como atractivo turístico y el uso de los medios de transporte para asistir a los eventos.

Se tiene que las actividades deportivas invernales en países donde el esquiar, el uso de funiculares, equipos motorizados, entre otros; no tendrían la demanda turística si no se contara con el medio de transporte. Para el turismo de sol y playa como son los deportes acuáticos en sus distintas modalidades; el turismo de aventura en la mayoría de los casos está vinculado al traslado en vehículos rústicos cuando es terrestre y aérea cuando se emplean medios de transportación como avionetas, helicópteros u otros equipos aerodinámicos.

De igual manera, esta la situación cuando el (los) medio(s) de transporte pasan hacer el atractivo turístico, tal como lo plantea (Cooper, et al, ob.cit: 548) "el transporte se convierte en un producto turístico atractivo por derecho en los casos de los cruceros, productos ferroviarios: el palacio sobre ruedas en la India, el tren azul en Sudáfrica, entre otros; productos marítimos como los viajes de un día en ferry por el mar Báltico y a través del canal de la Mancha". Se evidencia la relevancia de los medios de transporte al sector turismo y el uso de los mismos; la disposición de uso quedará sujeto a la necesidad del(los) turista(s) y de las empresas organizadoras encargadas de planificar los paquetes turísticos.

\section{TECNOLOGÍA DEL TRANSPORTE EN BENEFICIO DEL TURISMO.}

Establecida las relaciones entre el transporte y el turismo, se plantea la conexión de la tecnología del transporte para este sector de los viajes, el cual representa para muchos países una fuente de ingresos importantes para su economía. El cambio tecnológico en los viajes comenzó a raíz de la revolución industrial en el siglo XVIII cuando se inicia la construcción de canales de navegación para el transporte fluvial y la invención de los ferrocarriles; para el año 1840 se funda la primera agencia de viajes por el viajero Thomas Cook, empleando como medio de transporte los ferrocarriles que cubrían distancias cortas para la época; posteriormente en el siglo XIX “entre 1850 y 1873 Europa vivió un periodo de notable crecimiento económico vinculado con el auge de varias ramas productivas y al desarrollo del ferrocarril. A partir de 1873 el ritmo de crecimiento disminuyó y sólo 
pudo recuperarse en virtud de importantes avances en el área de la energía eléctrica y los motores, la química orgánica y los productos sintéticos, la maquina de combustión interna" (Nuñez, 2007:65).

Con la segunda guerra mundial en la aviación, se inicia una serie de transformaciones en los equipos aéreos existentes para la época, cuando surgen los desplazamientos de América del Norte a Europa y los aparatos aéreos requieren de mayor confort, mayor número de asientos, brindar servicios para viajes largos, entre otros aspectos; la revolución tecnológica y científica fue un delimitador del mejoramiento progresivo del sector turismo.

En la aviación las transformaciones tecnológicas han sido incuestionables, de comenzar con pequeños aviones para transportar sin comodidad a los pasajeros y a velocidades que permitían reducir el tiempo, hasta tener actualmente naves que surcan continentes a velocidades que sobrepasan las barreras del sonido como los concordes franceses (1971); aviones con un confort óptimo realizado esto a través de estudios ergonómicos, ofertando en sus áreas internas salas de esparcimiento, alimentos y bebidas, mayor cantidad de pasajeros caso los AIRBUS A380.

En el año 2009 la compañía Boeing presentó a las compañías aéreas su nuevo avión el 787-Dreamliner con características técnicas aeroespaciales eficiente, el cual transporta de 210 a 250 pasajeros, el cual recorre distancias superiores a los $14.000 \mathrm{Km}$; también, tiene como aspecto muy importante que minimiza el uso del combustible, de tal manera que la emisión de gases contaminantes afecta menos al ambiente y como punto especifico favorece el traslado del(los) pasajero(s) porque cuenta con comodidades internas que hacen confortables los viajes. Mientras algunos fabricantes buscan confort, otros buscan velocidad y se está proyectando la construcción de otros aviones de superior velocidad que el concorde francés, es decir, el campo tecnológico es cada vez más amplio para el mundo de los viajes.

En otro orden de ideas, es relevante explicar que los viajes aéreos característicos del turismo son los Charter son vuelos contratados con exclusividad, solicitados tanto por los pasajeros o por compañías turísticas con un precio a conveniencia de las partes, entre 
los aspectos relevantes de los vuelos Charter se tiene:

- No tienen horario, ni ruta regular establecida queda condicionado por el contratante.

- Se traslada a aeropuertos o pistas de aterrizaje en los cuales no pueden aterrizar vuelos regulares.

- La frecuencia de vuelos a un determinado lugar minimiza los precios a los pasajeros. Otro factor a considerar en los precios es que los costos de operación son menores.

- No están condicionados este tipo de servicio a registrarse en la I.AT.A, por lo tanto no quedan sujetos a regulación alguna por parte de la asociación. Sólo acatan las regulaciones aéreas de cada país.

Así como se ha realizado cambios sustanciales en la parte aérea, en lo correspondiente a lo acuático y terrestre también la tecnología ha contribuido de manera significativa al desarrollo del transporte; otro medio de transporte en el cual la ciencia y tecnología han incursionado y que actualmente ya se menciona como posibles viajes fuera de la atmósfera del planeta, son los viajes espaciales, a este tipo de viaje se les está denominado turismo espacial, naves que tendrán condiciones para efectuar viajes a la Luna, Marte, entre otros planetas; que están en estudio dentro de los avances tecnológico- científico del mundo de los viajes. En la primera década del siglo XXI se iniciaron los primeros vuelos turísticos espaciales a un costo muy elevado, sin embargo hay países dedicados a ofrecer los traslados dentro y fuera de la órbita del planeta con mayor número de días y con mejor confort.

El avance tecnológico-científico ha permitido que la frecuencia de traslados a distintas partes del mundo se haya incrementado considerablemente, al respecto se publicó en el barómetro mundial de viajes, lo siguiente: "las llegadas de turistas internacionales crecieron 5\% durante los primeros meses del 2011, consolidando así el repunte del 7\% registrado en el 2010. Según el Barómetro Mundial de la OMT el crecimiento fue positivo en todas las regiones y subregiones del mundo durante enero y febrero 2011, con excepción de Oriente Medio y el Norte de África" (Organización Mundial del Turismo, OMT, 2011:1). 
Para el sector turismo estos resultados presentados por la OMT representan un avance significativo, puesto que son indicadores del incremento de viajes que se ha generado por la versatilidad y disponibilidad de los medios de transporte para trasladar o desplazar a los turistas a diferentes para del mundo.

Como corolario del tema se puede apreciar, la influencia irrefutable que ha representado el cambio tecnológico-científico en los medios de transporte y lo que ha significado para los seres humanos estos avances; de esta manera, se establece la conexión y los beneficios que para el sector turismo, han representado los progresos de la tecnociencia.

\section{CONSIDERACIONES FINALES}

Históricamente los medios de transporte han sido parte fundamental del turismo, puesto que se requieren para el desplazamiento de los (el) turista(s) desde un punto de origen a un destino y es notable el desarrollo que en los mismos se ha venido generando por el avance tecnológico; en la medida que se han mejorando o perfeccionado los medios de transporte la demanda turística a crecido vertiginosamente. En el mundo de los viajes, los intercambios culturales, sociales, económicos, políticos, se producen porque existen los medios de transporte.

El sector turismo para formar parte de la economía de un país requiere de la interrelación de todas las empresas operadoras de bienes y servicios, de modo que el proceso de comercialización del producto turístico va a depender de la vinculación que se tenga entre las empresas minoristas, tour operadores y mayoristas, alojamiento, empresas de alimentos y bebidas, las de recreación y las empresas transportistas, entre otras.

Es indudable, que un factor determinante en el progreso tecnológico-científico se manifiesta en la calidad del servicio, seguridad, rapidez y confort de los medios de transportación que se ofrecen a los turistas, porque para realizar los recorridos en transportes públicos o privados los mismos deben al menos cumplir con los requisitos no solamente básicos sino con otras exigencias que en el mundo actual son condicionantes para satisfacer las necesidades o expectativas de los viajeros sobre todo en el sector turismo. 
Con referencia al crecimiento tecnológico-científico es importante destacar que en la transportación aérea se ha notado cambios sustanciales, porque se evidencian cuando las líneas comerciales utilizan aviones propulsión a chorro, hay mejoramiento en el servicio, disminución en los precios de los pasajes e incluso a ser más eficientes en el consumo de combustibles con energías alternativas o disminución de combustibles para no generar impacto ambiental, en el aumento de la capacidad de pasajeros y carga, las distancias y frecuencias entre vuelos se han reducido. Este avance se confirma también en el transporte acuático especialmente con los cruceros que están equipados con los últimos adelantos tecnológicos, en el transporte terrestre con la diversidad de los mismos y el transporte espacial, en el cual los científicos y tecnólogos se han abocado progresivamente para buscar otros medios de desplazamiento sobre todo en el sector turístico.

En resumen, se plantea "de manera creciente, la frontera entre transporte y entretenimiento se disuelve a medida que la travesía a la embarcación se convierte en un fin y no sólo un medio de transporte" (Holloway, 1997:146).

\section{BIBLIOGRAFÍA}

Boeing. (2011). El 787 Dreamliner de Boeing dará Nuevas Soluciones a las Aerolíneas y Pasajeros. Disponible: http://www.boeing.es/ViewContent.do?id=1481 [Consulta: 2010, Diciembre 05]

Cooper,Chris, Fletcher,Jhon, Fyall,Alan, Gilbert,David y Wanhill, Stephen. (2007). El turismo. Teoría y Práctica, España, Síntesis.

Cuervo, Raimundo (1967) El turismo como medio de comunicación humana, México, Departamento de Turismo.

De La Torre Padilla, Oscar (1980). Turismo- fenómeno social, México, Fondo de Cultura Económica.

Holloway, John Chistopher.(1997). El Negocio del turismo, México, Diana.

Hunziker, Walter y Krapf, Kurt (1942). Grundiss der Allgemeire Fremderverkehrslebre, Zurich, Plygraphicher verlag.

Leiper, Neil (1979). The framework of tourism: Towards a definition of tourism, tourist and the tourism industry. Annals of Tourism Research, vol. 4, no 6, pp.309407.

Nuñez, Jorge (2007). La Ciencia y la tecnología como procesos sociales, Cuba, Félix Varela.

Organización Mundial de Turismo (OMT) (2011). Barómetro Mundial del Turismo. 
[Datos en línea]. Disponible: http://www.unwto.org/facts/eng/barometer. htm [Consulta: 2010, Diciembre 05]

Taylor, John \& Swanborough, Gordon (1977) Aviación civil del mundo actual, Madrid, San Martín

Recibido: 04/03/2012

Aceptado: 11/06/2012

Arbitrado anónimamente 